\title{
Analysis of Food quality and Food Adulterants from Different Departmental \& Local Grocery Stores by Qualitative Analysis for Food Safety
}

\author{
Shruti Awasthi**, Kirti Jain**, Anwesha Das*, Raza Alam*, Ganesh Surti*, \\ Kishan N. \\ Department Of Biochemistry, Garden City College, Bangalore.
}

\begin{abstract}
Food is one of the basic needs for every living being and very important aspect for life. But now day's foods are affected by different adulterants. Adulteration is a substance which reduces the vital importance of food. Some of the common adulterants are mineral oils, argemone oils, castor oils in edible oils; vanaspathi, mashed potato in ghee; invert sugar or jiggery in honey, kesari dal, lead chromate in pulses etc. which can leads to Epidemic dropsy, Glaucoma, Cardiac arrest, Lathyrism (crippling spastic paraplegia), Anemia, abortion, paralysis, brain damage, cancer etc.

The aim is to evaluate the presence of adulterant from daily uses food materials like Milk, Butter, Edible oil, Honey, Pulses, Wheat Flour, Black Pepper, Chilli powder, Coffee powder which we collected from different departmental and local grocery stores and checked the presence of individual adulterants by biochemical qualitative analysis. The colour change of the sample indicate the according to the reagents is indicates the presence of different adulterants. This information can help to grow the food safety and also people can be aware about the food brands for a healthy life.
\end{abstract}

Keywords: Food, adulterants, Milk, Butter, Edible oil, Honey, Pulses, Wheat Flour, Black Pepper, Chilli powder, Coffee powder.

\section{Introduction}

Food is any substance, composed of carbohydrates, water, fats and proteins, which can be eaten or drunk by animals, including humans, for nutrition or pleasure. ${ }^{(1)}$ By other words food is one of the basic needs for every living being and very important aspect for life. Many plants or plant parts are eaten as food. There are around 2,000 plant species which are cultivated for food, and many have several distinct cultivars. Almost all foods are of plant or animal origin. Cereal grain is a staple food that provides more food energy worldwide than any other type of crop. ${ }^{(2)}$ Maize, wheat, and rice together account for $87 \%$ of all grain production worldwide. ${ }^{(3)}$ Animals are also used as food either directly or indirectly by the products they produce. Meat is an example of a direct product taken from an animal, which comes from either muscle systems or from organs. Food products produced by animals include milk produced by mammary glands, which in many cultures is drunk or processed into dairy products such as cheese or butter. In addition, birds and other animals lay eggs, which are often eaten, and bees produce honey, reduced nectar from flowers, which is a popular sweetener in many cultures. ${ }^{(4)}$

Food can be contaminated by different adulterants. Adulterants are chemical substances which should not be contained within other substances (e.g. food, beverages, fuels for legal or other reasons). The addition of adulterants is called adulteration. ${ }^{(5)}$ It is also a substance which also reduces the vital importance of food and causes some toxic effect to the human body. Adulterants when used in illicit drugs are called cutting agents, while deliberate addition of toxic adulterants to food or other products for human consumption is known as poisoning. ${ }^{(6)}$ Adulteration in food is normally present in its most crude form. Adulterants may be intentionally added to more expensive substances to increase visible quantities and reduce manufacturing costs, or for some other deceptive or malicious purpose. Adulterants may also be accidentally or unknowingly introduced into substances. In India normally the contamination/adulteration in food is done either for financial gain or due to carelessness and lack in proper hygienic condition of processing, storing, transportation and marketing. This ultimately results that the consumer is either cheated or often become victim of diseases. Such types of adulteration are quite common in developing countries or backward countries. Some of the common adulterants are mineral oils, argemone oils, castor oils in edible oils; vanaspathi, mashed potato in ghee; invert sugar or jiggery in honey, kesari dal, lead chromate in pulses etc. which can leads to Epidemic dropsy, Glaucoma, Cardiac arrest, Lathyrism (crippling spastic paraplegia), Anemia, abortion, paralysis, brain damage, cancer etc.

\section{Materials \& Methods}

Food materials like Milk, Butter, Mustered oil, Honey, Pulses, Wheat Flour, Black Pepper, Chilli powder, Coffee powder which we collected from different departmental and local grocery stores. Some reagents 
like Iodine reagent, Concentrated $\mathrm{HCl}$, Sucrose, $0.5 \mathrm{~N}$ ethanolic $\mathrm{KOH}$, Concentrated $\mathrm{HNO}_{3}$, Solvent ether, Resorcinol, Carbon tetra-chloride $\left(\mathrm{CCl}_{4}\right)$, Chloroform were collected which were used for biochemical tests.

Qualitative tests are done for detecting presence of adulterants. We prepared certain reagents according to the sample for qualitative analysis to observe the color change or the appearance of the sample according to adulterants presents in the sample. Adulterants milk will show blue colour with prepared Iodine reagents, adulterants butter will show maroon colour with concentrated $\mathrm{HCl}$ and pinch of sucrose, adulterant edible oil will appear turbid and will separate, yellow, orange or crimson colour lower acid layer with prepared reagents for two kind of adulteration, adulterants honey will turn to cherry red colour with prepared reagents, adulterants honey will turn to cherry red colour with prepared reagents, adulterants pulses will turn to pink colour immediately with prepared reagents, adulterants of wheat, Chili powder, coffee powder and black pepper will settled down or will float on the surface of the prepared reagents and water.

\section{Result \& Discussion}

The obtained results of the taken samples are tabulated below-

\begin{tabular}{|c|c|c|}
\hline Food Materials & Brand Name & Result \\
\hline \multirow[t]{2}{*}{ Butter } & Sample A & Sample colour does not change into maroon. (fig-2.B)The \\
\hline & Sample B & Sample colour changes in to maroon. (fig-2.A) \\
\hline \multirow[t]{2}{*}{ Milk } & Sample A & The colour of the does not change into blue. (fig-1.B) \\
\hline & Sample B & colour of the does not change into blue. (fig-1.A ) \\
\hline \multirow[t]{2}{*}{ Edible oil } & Sample A & $\begin{array}{l}\text { Shows reddish brown colour of the acid layer and turbidity. (fig-3.A \& } \\
\text { 4.A) }\end{array}$ \\
\hline & Sample B & $\begin{array}{l}\text { Shows reddish brown colour of the acid layer and turbidity. (fig-3.B \& } \\
\text { 4.B) }\end{array}$ \\
\hline \multirow[t]{2}{*}{ Honey } & Sample A & Change of colour in cherry red. (fig-5.A) \\
\hline & Sample B & No change of colour as cherry red. (fig-5.B) \\
\hline \multirow[t]{2}{*}{ Pulses } & Sample A & Change of colour in pink. (fig- 6. A\& 7.A) \\
\hline & Sample B & Less change of colour into pink (fig- 6.B \& 7.B) \\
\hline \multirow[t]{2}{*}{ Wheat } & Sample A & Bran is not floating on the surface of the water. (fig- 8.A) \\
\hline & Sample B & Bran is floating on the surface of the water.(fig- 8.B) \\
\hline \multirow[t]{2}{*}{ Black paper } & Sample A & Papaya seed is floating upon the CCl4 solution. (fig- 9.A) \\
\hline & Sample B & Papaya seed is not floating upon the CCl4 solution. (fig- 9.B) \\
\hline \multirow[t]{2}{*}{ Chilly powder } & Sample A & Adulterants settled down. (fig- 10.A) \\
\hline & Sample B & No adulterants settled down. (fig- 10.B) \\
\hline \multirow[t]{2}{*}{ Coffee } & Sample A & Floating of chicory onto the water surface. (fig- 11.A) \\
\hline & Sample B & No floating of chicory onto the water surface. (fig- 11.B) \\
\hline
\end{tabular}

i. Milk: - Two milk sample that is 1. Raw milk from stable 2. Good life milk from M.K. Retailer departmental was collected and tests for adulteration such as starch. By this test none of the milk sample was found to be adulterated. Starch is common adulterants in milk and milk is very basic food for growth of infants and children. A recent study of starch says that starch is interfering in the growth of human. It also causes Stomach disorder.

ii. Butter: - Two renowned brands were chosen for butter 1. Amul 2. Nutralite and checked for adulteration. The Amul butter sample was change its colour to maroon after test which indicates presence of hydrogenated fat where as the Nutralite butter does not change its colour. That proves Amul butter is adulterated with hydrogenated fat. Adulterants for butter which cause increase level of bad cholesterol results accumulation of excess fat, high blood pressure, heart attack etc.

iii. Mustard oil: - Two categories 1. From local grocery stores 2. Dhara oil were collected and checked for two kind of adulteration i.e. argemone oil and mineral oil. Both the samples had shown the turbidity which means both the sample contain mineral oil. But the local grocery store oil sample shows more turbidity than MK Retailer sample. Mostly mix with edible oils mixture of white oil, petroleum fraction causes Damage to liver, \& has carcinogenic effects.

Next the oil samples were checked for argemone oil contamination. Here also both the samples contain argemone oil contamination. But the oil from local grocery shop completely turns into crimson red. This indicates more amount of argemone oil present in the sample where the MK retailer oil sample changes to yellow colour means less amount of argemone oil is present. Mostly mixed with mustered oil causes' Epidemic dropsy, Glaucoma, Cardiac arrest, Loss of eyesight, heart diseases, and tumor. 
iv. Honey: - Two honey samples such as 1. Dabur honey 2. From local grocery shop. The local grocery store honey changes its colour to cherry red which indicates adulteration with inverted sugar/jiggery and Dabur honey is not adulterated. Adulterants for honey. Inverted sugar or jaggery has no deleterious effect on human being. But as we know that honey has antibacterial effect upon common cold and to some digestive problem, the mixture of inverted sugar or jaggery can restrict antibacterial properties of honey sometimes also causes Stomach disorder.

v. Wheat flour: - Wheat flour samples were collected form 1. Local grocery store and 2. Mk Retailer and sprinkled on the water surface and checked for the floating brans. Here in MK retailer sample floating bran was found where no floating brans have been found in local grocery store wheat flour sample. Which means the MK retailer sample was adulterated and the local grocery shop sample is not adulterated. Mostly found in wheat flour. Limited level of bran can control cholesterol level but exceed the limit of bran can cause lowering absorption of calcium, magnesium and other minerals.

vi. Pluses: - Two pulses sample from 1. Local grocery shop and 2. MK retailer. Pulses were checked for two adulterants. First one is Metanil Yellow (dye) where both the samples show the pink colour which means both the samples was adulterated. It is used paint \& textile companies; it is most bad adulterants which mostly mixed with pulses, turmeric powder \& also it is toxic and highly carcinogenic.

Next test was done for presence of lead chromate same as by adding concentrated $\mathrm{HCl}$ to pulses water and checked for the colour change to pink. Here also both the samples shown pink colouration, which means both the samples were adulterated with lad chromate. It is a dye mixed with chilly, pulses and has dangerous effect like Anemia, abortion, paralysis, brain damage etc.

vii. Black pepper: - Two samples from 1 . Local grocery shop 2 . MK retailer were collected \& checked by putting black pepper in the $\mathrm{CCl}_{4}$ solution where adulteration papaya seeds will be floating on the water surface and black pepper will be settled down. Here in MK retailer sample no adulteration were found but adulteration were found in local grocery sample, means local grocery shop sample was found to adulterated and MK retailer sample is not adulterated. Mixed with black pepper it's not only lowering the quality of black pepper it also cause some stomach disorders.

viii. Chilli powder: - Two samples from 1. Local grocery shop 2. MK retailer was collected and checked for adulteration. Here in Mk retailer sample no red brick and dirt were settled down but in local grocery shop sample was found to be adulterated with red brick and dirt. The MK retailer sample was not adulterated. It adulterants mixed with chili powder causes Damage of digestive tract \& stomach disorders.

ix. Coffee powder: - Two renowned coffee samples were collected 1. Nescafe \& 2. Coffee day coffee powder. In the Nescafe sample no floating chicory was found so this sample is not adulterated but in the coffee day coffee powder floating chicory was found which means this sample is adulterated with chicory. Mixed with a famous beverages coffee cause Stomach disorder, Giddiness and joint pain.

\section{Conclusion}

From the above findings and discussions lastly we can conclude that the adulteration which mixes in the food items can cause tremendous effect on health without our knowledge. This can be prevented by few alerting steps of our society. The government should make a check on hike of price. Selection of wholesome and non-adulterated food is essential for daily life to make sure that such foods do not cause any health hazard. Although it is not possible to ensure the adulterants in wholesome food only on visual examination when the toxic contaminants are present in ppm/ppb level. Government bodies should inspect food quality of both the brands and local stores. However, visual examination of the food before purchase makes sure to ensure absence of insects, visual fungus, foreign matters, etc. Consumer should be able to judge what is good and what is not. The consumer should avoid taking food from an unhygienic place and food being prepared under unhygienic conditions. Label declaration on packed food is very important for knowing the ingredients and nutritional value. Government should aware people about adulteration in food and there adverse effects. Expiry dates should be checked prior to buy something. Avoid low quality local grocery food item just to save few penny. Buy the item with a proper bar code billing. Inform and complain to the consumer forums about the shops if such deleterious adulterants would found in food items.

Business oriented people just forgot the humanity in behind of money making mentality. The above general consciousnesses are very easy to initiate for our healthy life. If we actively take part in changes at least for our own concern then we bring about a healthy and non hazardous future for our forth coming offspring.

[1]. Encyclopedia Britannica definition

\section{References}

[2]. McGee, 333-334

[3]. McGee, Chapter 9

[4]. Davidson, 81-82

[5]. Weise, Elizabeth (April 24, 2007). "Food tests promise tough task for FDA". USA Today. Retrieved 2007-04-29

[6]. The fight against food adulteration, Noel G Coley, RSC, Education in chemistry, Issues, Mar 2005 


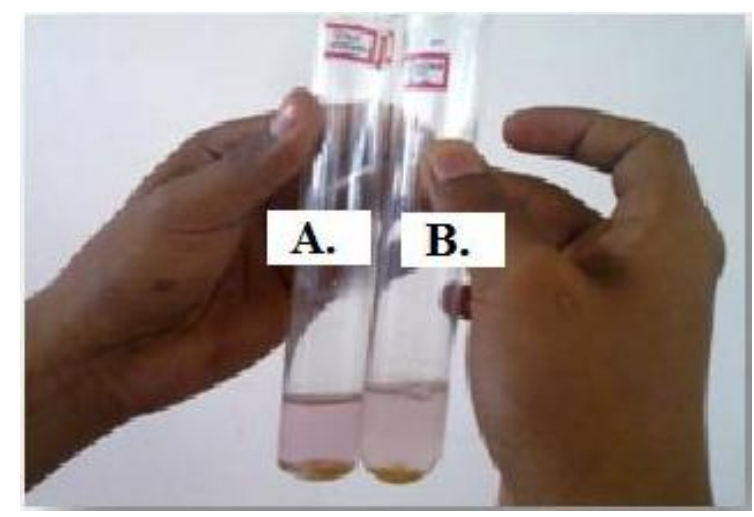

Fig- 1

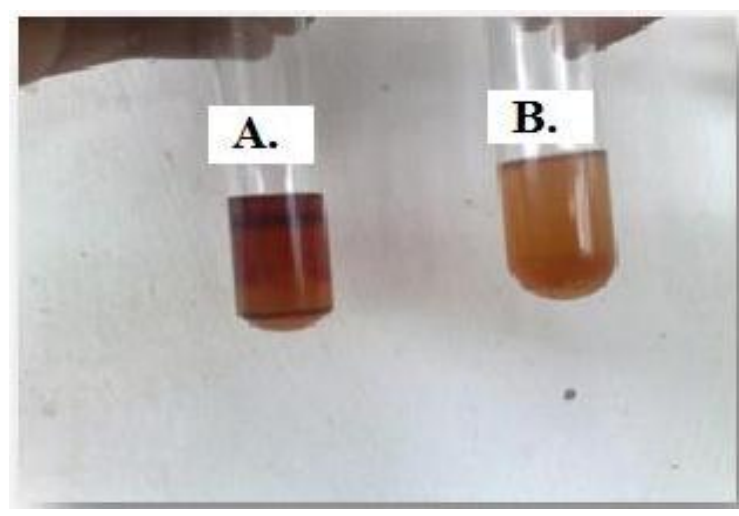

Fig- 3

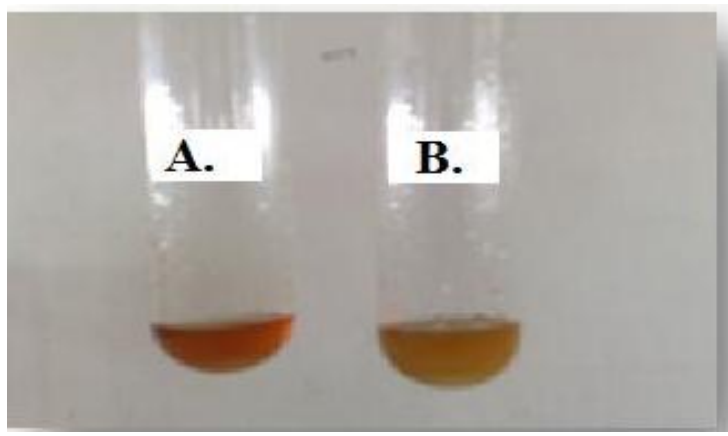

Fig- 5

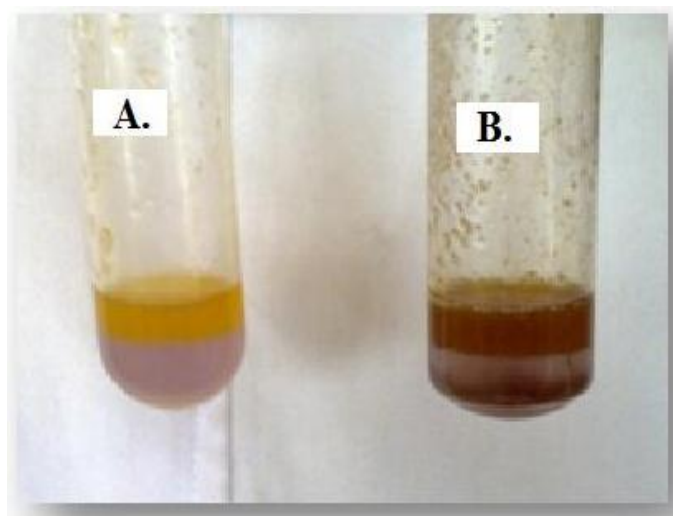

Fig- 7

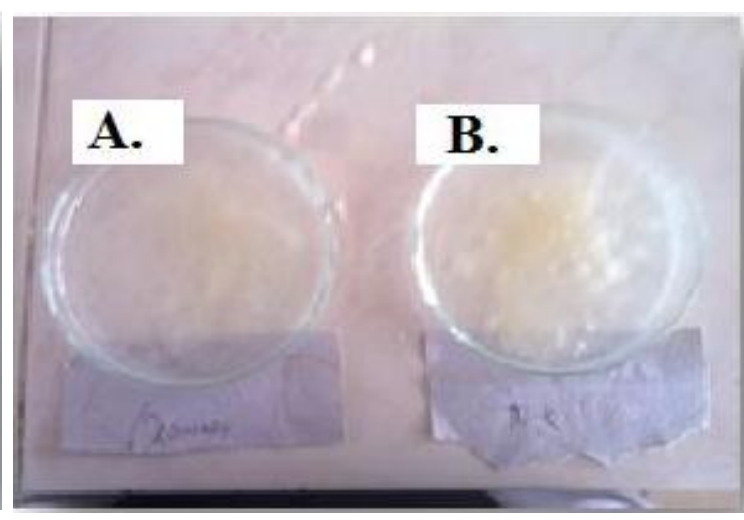

Fig- 2

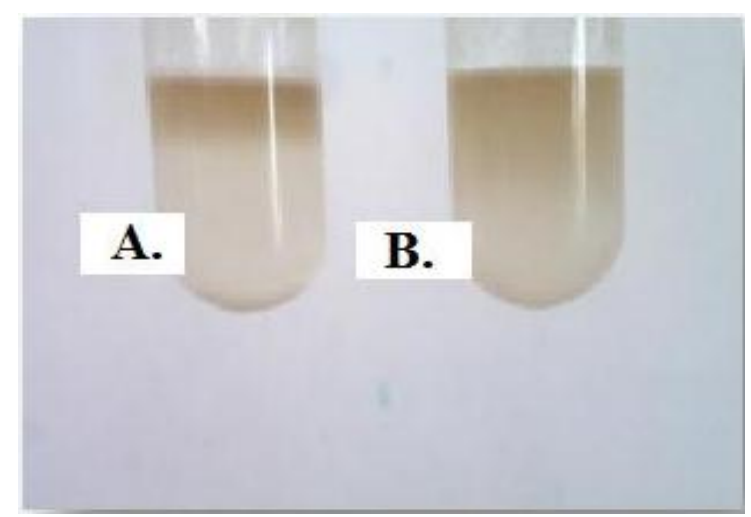

Fig- 4

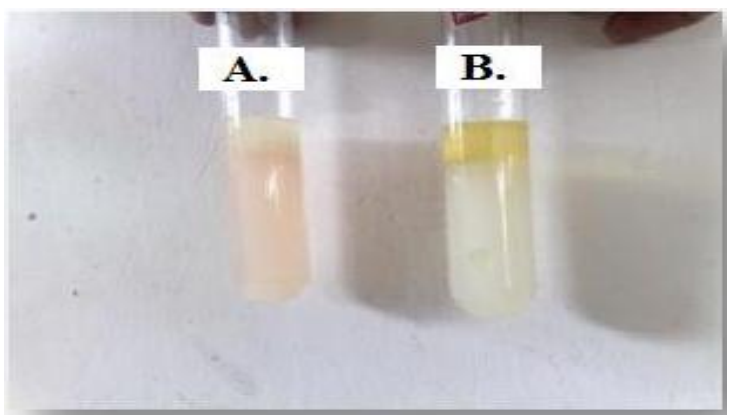

Fig- 6

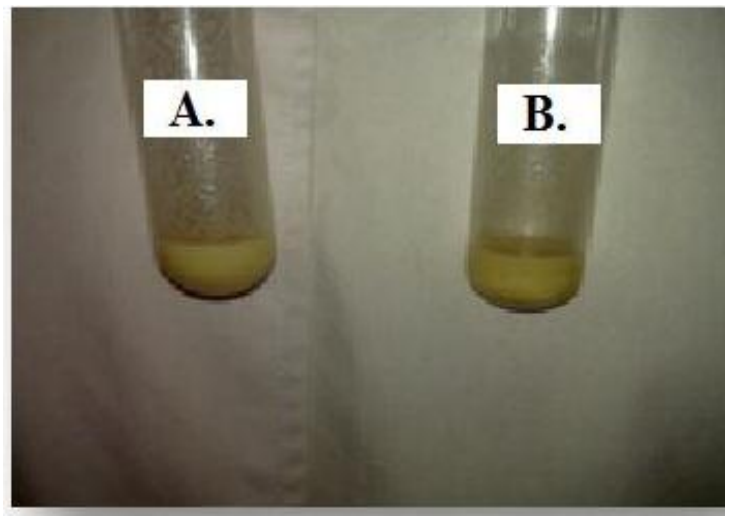

Fig- 8 


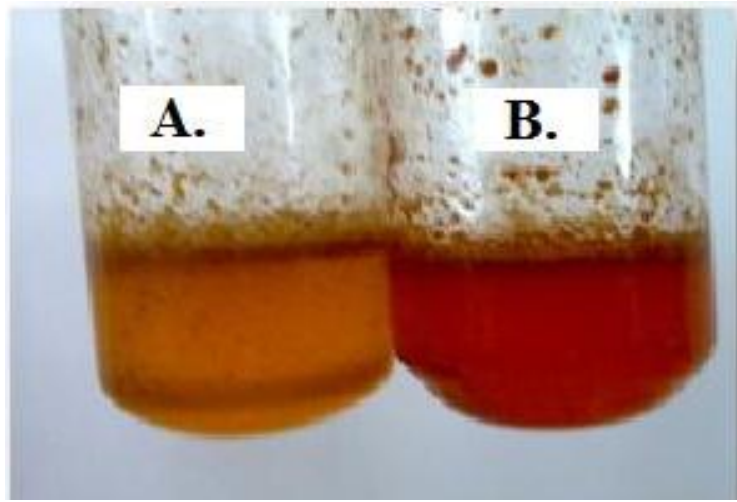

Fig- 9

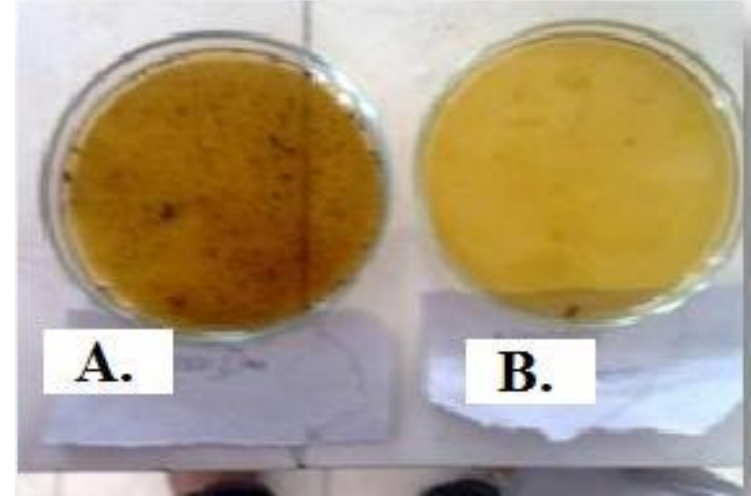

Fig- 10

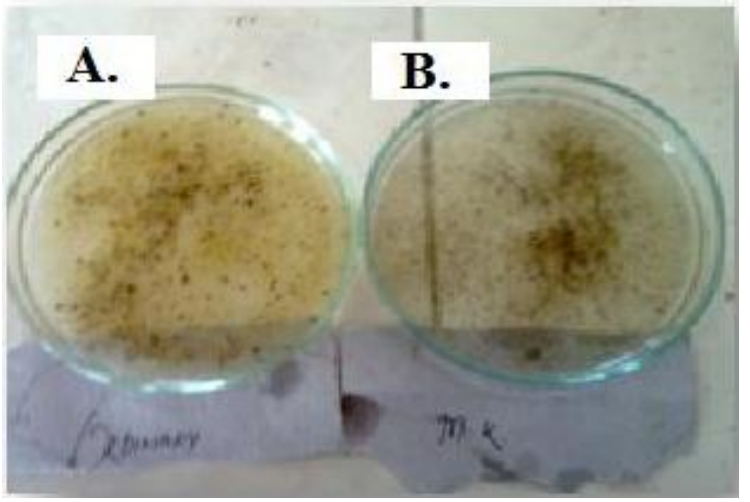

Fig- 11

\section{Legend:}

Fig- 1: Butter Sample -A. Nutralite \& B. Amul butter respectively. Amul butter is adulterated.

Fig- 2: Milk Sample - A. Raw milk from stable \& B. good life milk respectively. No adulteration in both Samples.

Fig- 3: Edible oil: (Argemone Oil)- A. Oil from local grocery store \& B. Dhara oil from MK retailer respectively. Both samples shows adulteration present of Argemone oil but adulteration in local grocery store is more.

Fig -4: Edible oil: (Mineral Oil)- A. Oil from local grocery store \& B. Dhara oil from MK retailer respectively. Both samples shows adulteration present of Mineral oil but adulteration in local grocery store is more.

Fig -5: Honey - A. Honey from local grocery store \& B. Dabur honey respectively. Honey from local grocery store is adulterated.

Fig -6: Pulses: [Metanil Yellow (dye)] - A. Pulses from local grocery store \& B. MK retailer respectively. Both samples shows adulteration but adulteration in local grocery store is more.

Fig -7: Pulses: (Lead Chromate) - A. Pulses from local grocery store \& B. MK retailer respectively. Both samples shows adulteration but adulteration in local grocery store is more.

Fig- 8: Wheat Sample- A. Wheat from local grocery store \& B. MK retailer respectively. Wheat form MK retailer is adulterated.

Fig -9: Black Pepper- A. Black pepper from local grocery store \& B. MK retailer respectively. Black pepper from local grocery store is adulterated.

Fig -10: Chilli powder- A. Chilli powder from local grocery store \& B. MK retailer respectively. Chilli powder from local grocery store is adulterated.

Fig -11: Coffee Sample - A. Coffee day \& B. Nescafe respectively. Coffee day coffee powder is adulterated. 